\title{
MARKETING PROFISSIONAL E VISIBILIDADE SOCIAL NA ENFERMAGEM: UMA ESTRATÉGIA DE VALORIZAÇÃO DE RECURSOS HUMANOS $^{1}$
}

\author{
Isabel Amélia Costa Mendes², Maria Auxiliadora Trevizan³, Alessandra Mazzo4, Simone de Godoy5, Carla \\ Aparecida Arena Ventura 6
}

\footnotetext{
${ }^{1}$ Conferência proferida no $62^{\circ}$ Congresso Brasileiro de Enfermagem, a convite da Associação Brasileira de Enfermagem.

${ }^{2}$ Doutora em Enfermagem. Professora Titular do Departamento de Enfermagem Geral e Especializada (DEGE) da Escola de Enfermagem de Ribeirão Preto (EERP) da Universidade de São Paulo (USP). São Paulo, Brasil. E-mail: iamendes@usp.br

${ }^{3}$ Doutora em Enfermagem. Professora Titular do DEGE/EERP/USP. São Paulo, Brasil. E-mail: trevizan@eerp.usp.br

${ }^{4}$ Doutora em Enfermagem. Professora Doutora do DEGE/EERP/USP. São Paulo, Brasil. E-mail: amazzo@eerp.usp.br

${ }^{5}$ Doutora em Ciências. Especialista em Laboratório do DEGE/EERP/USP. São Paulo, Brasil. E-mail: sig@eerp.usp.br

${ }^{6}$ Doutora em Administração. Professora Doutora do Departamento de Enfermagem Psiquiátrica e Ciências Humanas da EERP/ USP. São Paulo, Brasil. E-mail: caaventu@eerp.usp.br
}

RESUMO: No cenário internacional percebe-se um movimento de valorização da enfermagem entre as profissões de saúde, utilizando, para tal, ferramentas de marketing profissional. Por meio de revisão integrativa da literatura discutimos o marketing como ferramenta para o incremento da visibilidade da enfermagem. Utilizamos as bases LILACS, MEDLINE, Web of Science e SciELO. Foram selecionadas publicações que relacionavam o marketing à enfermagem, no período de 2000 a 2010. Encontramos 91 artigos e, após leitura exaustiva dos títulos e resumos, foram selecionadas oito publicações que abordaram, em sua maioria, o uso dos meios de comunicação em massa como estratégia de marketing da profissão para promoção de saúde e imagem profissional veiculada em meios como a internet e a televisão. O tema é pouco explorado na pesquisa em enfermagem, mas há perspectivas de avanços, pelo interesse que desperta e pelo reconhecimento crescente de sua importância.

DESCRITORES: Enfermagem. Marketing. Saúde. Recursos humanos.

\section{PROFESSIONAL MARKETING AND SOCIAL VISIBILITY IN NURSING: A STRATEGY TO VALUE HUMAN RESOURCES}

\begin{abstract}
There is a movement within the international scenario to value nursing among other health professions using professional marketing tools. Through an integrative literature review using the LILACS, MEDLINE, Web of Science, and SciELO databases, we discuss marketing as a tool for increasing Nursing visibility. As a result, publications relating marketing to Nursing published in the period from 2000 to 2010 were selected. After reading the titles and abstracts of the 91 articles resulted from the database searches, eight articles which outlined in its majority the use of means of mass communication as a professional marketing strategy for health promotion and a professional image linked to media such as the internet and television were selected. The theme is not well explored in nursing research, but there are perspectives for improvement, due to the increasing interest and recognition of its importance.
\end{abstract}

DESCRIPTORS: Nursing. Marketing. Health. Human resources.

\section{MARKETING PROFESIONAL Y VISIBILIDAD SOCIAL EN ENFERMERÍA: UNA ESTRATEGIA DE VALORIZACIÓN DE RECURSOS HUMANOS}

RESUMEN: En el ámbito internacional hay un movimiento de valorización de la enfermería entre las profesiones de salud, que usa para ello, herramientas de marketing profesional. Por medio de una revisión integradora de la literatura, se discutió el marketing como herramienta para el incremento de la visibilidad de la enfermería. Se utilizaron las bases LILACS, MEDLINE, Web of Science y SciELO. Se seleccionaron publicaciones que asociaban el marketing a la enfermería en el período de 2000 a 2010. Se encontraron 91 artículos, y después de la lectura exhaustiva de los títulos y resúmenes se seleccionaron ocho publicaciones que abordan, en su mayoría, el uso de los medios de comunicación como estrategia de marketing de la profesión para promover la salud y la imagen profesional transmitida en medios como la internet y televisión. El tema es poco explorado en la investigación en enfermería, pero hay perspectivas de avance por el interés que despierta y por el reconocimiento creciente de su importancia.

DESCRIPTORES: Enfermería. Mercadeo. Salud. Recursos humanos. 


\section{INTRODUÇÃO}

O cenário atual dos serviços de saúde demonstra uma demanda crescente de pacientes em busca de cuidados prestados pelos profissionais da área; todavia, sem o aporte equivalente de recursos financeiros, as organizações decidem reduzir o número de colaboradores da enfermagem.

Os administradores de serviços da saúde utilizam meios que garantem a redução dos custos operacionais dos serviços prestados, reduzindo também a permanência do paciente sob regime de internação, além de aumentar o número de pacientes por enfermeiro. Ao mesmo tempo, os consumidores estão preocupados e atentos aos erros, aos seus direitos à assistência com qualidade, e procuram por serviços de saúde que estejam mais focados na família, e que ofereçam acessibilidade fácil, conveniência, privacidade e garantia de serviços tecnicamente avançados, seguros e eficientes.

Os profissionais de enfermagem, por sua vez, procuram por melhores ambientes de trabalho nos quais a proporção paciente/enfermeiro seja propícia ao desempenho de suas funções, com satisfação e segurança.

Há uma dicotomia entre o ambiente/condições de trabalho desejados pelos enfermeiros e as políticas adotadas pelas instituições hospitalares que têm fins lucrativos e, ainda, as dificuldades logísticas das instituições sem fins lucrativos. Adicionalmente, há escassez de estudos que permitam analisar em profundidade o modo como os recursos humanos de saúde podem influenciar o sucesso das reformas implementadas no setor. ${ }^{1}$

Deste contexto resultam indicadores mais preocupantes: aumento de erros praticados por profissionais de saúde, custos trabalhistas, ações sindicais, insatisfação dos profissionais com baixa valorização, além de elevado custo de novas tecnologias. O surgimento de novas doenças, a natureza precária de trabalho, desemprego, pobreza e crescimento demográfico requerem melhorias na eficiência e na eficácia dos sistemas de saúde que, por sua vez, exigem a gestão formal de recursos humanos; fator este que não foi suficientemente abordado durante as reformas. ${ }^{2}$

Considerando este difícil cenário, emerge a questão sobre a situação da enfermagem nestas circunstâncias. No mundo há hoje 17,5 milhões de enfermeiros e obstetrizes; o déficit desses profissionais é grande e variável por país, em todas as regiões do globo. ${ }^{3}$

Tanto nos países desenvolvidos, como nos países em desenvolvimento, o déficit atual e futuro de enfermeiros constitui um problema crucial com a perspectiva de ameaça aos sistemas nacionais de saúde. No Brasil, temos 1,5 milhão de profissionais de enfermagem, e a escassez aqui, como em todos os países, deve ser enfrentada estrategicamente, de acordo com a realidade e capacidade de solução local. ${ }^{3}$

Mas, seja qual for o país ou o seu contexto, é inevitável que se enfrente o problema com duas ações programáticas complementares, aplicadas de forma contínua e consideradas como prioritárias, entre outras adotadas: 1) a atração de novos talentos e vocações para a profissão; 2) a aplicação de estratégias e mecanismos de retenção dos profissionais na profissão.

Só com políticas nacionais de valorização do profissional de enfermagem (pelas agências empregadoras, pela sociedade, pela equipe de saúde e pela própria profissão de enfermagem) é que poderemos contar com o comprometimento do enfermeiro para com sua profissão. Sem políticas de valorização, o profissional se desmotiva e migra para outra ocupação. Este é um ponto importante e fundamental para lidarmos com a necessidade de aumento dos índices de retenção.

Outro ponto que requer atenção por parte dos órgãos governamentais e reguladores da profissãoé o fenômeno da migração. Como o déficit nos países desenvolvidos já é considerável e a perspectiva de acentuação é também elevada, pela baixa demanda de seus cidadãos aos cursos de enfermagem por eles oferecidos, outro agravante que se adiciona aos países em desenvolvimento é o da migração de seus profissionais. Se nesses países a valorização é pequena, o profissional acaba seduzido por melhores condições de trabalho e de vida a eles oferecido.

Os governos estão cientes de que os sistemas de saúde somente serão viabilizados se houver uma enfermagem suficientemente forte. Segundo a diretora da Organização Mundial da Saúde (OMS), " [...] a enfermagem constitui a espinha dorsal dos sistemas de saúde" ${ }^{4: 67}$ Este conceito é repetidamente registrado na documentação oficial deste organismo internacional e transmitido para os governantes e ministros da saúde dos países membros da Organização das Nações Unidas (ONU).

As Resoluções da OMS constituem peças-chave de orientação aos países membros na implementação de políticas e estratégias de saúde. Existe o reconhecimento explícito da importância da enfermagem para o sucesso das políticas adotadas nas World Health Assemblies (WHA), como ilustrado pelas seguintes resoluções de: 1977 (WHA 30.48, 
que trata do papel do pessoal de enfermagem e obstetrícia nos cuidados primários de saúde, bem como as de 1983, WHA 36.11; 1989, WHA 42.27; 1992, WHA 45.5; 1996, WHA 49.1 e 2001, WHA 54.12 , as quais enfatizam o fortalecimento da enfermagem e obstetrícia na estratégia de saúde para todos. ${ }^{5-13}$ Todas essas resoluções exaltam:

1) a necessidade premente de aumentar o número de escolas e programas de treinamento de professores e gestores de serviços de enfermagem;

2) a necessidade de se desenvolver líderes para motivar e estimular as mudanças requeridas para orientar a educação e a prática de enfermagem;

3) as recomendações aos governos para que revejam suas necessidades nacionais de recursos de enfermagem, que adotem medidas para evitar escassez no futuro, bem como para que operacionalizem estratégias de recrutamento, formação, retenção, educação permanente e melhoria contínua nas qualificações dos profissionais de enfermagem, para que se possa atender às necessidades nacionais;

4) o encorajamento e o apoio à contratação de profissionais de enfermagem e obstetrícia para posições de liderança e gestão, facilitando a participação das mesmas no planejamento e implementação das atividades de saúde dos países.

A Assembléia de 2006 incitou os governos a confirmarem seu compromisso de fortalecer a enfermagem e obstetrícia e registrou requerimento ao Diretor Geral da OMS, solicitando que encoraje o apoio dos estados-membros na provisão de ambientes de trabalho que sejam seguros, que promovam a retenção de enfermeiros e obstetrizes e que apóiem os estados-membros no fortalecimento e na aplicação de regras de recrutamento ético. ${ }^{14}$ Tais documentos constituem evidência de que os sistemas de saúde são monitorados e cobrados (pelo organismo internacional ao qual são vinculados) para que valorizem a enfermagem e seus profissionais.

Partindo do pressuposto de que a inabilidade da profissão valorizar seus profissionais ameaça a sua própria visibilidade, entendemos que a enfermagem precisa ter um posicionamento mais pró-ativo neste cenário, aliando-se ao apoio de organismos internacionais e valendo-se de evidências para reivindicar melhores condições de trabalho e mais valorização, por um lado; de outro lado, deve mostrar o seu valor e sua competência para o sistema, para seus clientes, para as instituições e para a sociedade, ou seja, usar o marketing profissional, alcançando maior visibilidade social, através do resultado de seu trabalho.
O marketing é uma das mais importantes estratégias passíveis de favorecer a visibilidade de uma profissão. Para compreender marketing profissional é necessário agregar os conceitos do marketing à carreira, bem como ao desenvolvimento de produtos, sejam eles bens ou de serviços. Quando se trata de serviços, o marketing deve estar focalizado nas credenciais do profissional que são, fundamentalmente, a qualidade de seu trabalho, seu talento e sua disposição. ${ }^{15}$

Nesse sentido, é necessário que o profissional utilize seu marketing pessoal, o que consiste na venda do produto "você", construído pela imagem positiva de si para si mesmo e para as pessoas. Para construir sua imagem e trabalhar o marketing pessoal, o indivíduo precisa ser executivo chefe de si mesmo. É pensar em você enquanto uma empresa que necessita de uma marca para ser vendida. Essa marca deve "encantar o cliente e deixar claro qual benefício lhe trará". 16:80 Portanto, é preciso reconhecer as necessidades de seus clientes, desenvolvendo-se enquanto profissional, estabelecendo e cultivando uma rede de relacionamentos que lhe propiciem as melhores oportunidades do mercado, de maneira ética e empreendedora. ${ }^{16}$

Esse trabalho teve como objetivo discutir o marketing como ferramenta para o incremento da visibilidade da enfermagem, com base em registros da literatura.

A prática de enfermagem tem se dado de maneira evolutiva, mas sem a visibilidade necessária. Alguns estudos demonstram que a sociedade não reconhece a profissão, o que provoca baixa procura pelos cursos de enfermagem, diminuta valorização do produto de seu trabalho, dificuldades relacionadas ao poder dentro das instituições. Justifica-se, portanto, este trabalho, uma vez que a enfermagem não se dedica ao seu marketing profissional e os enfermeiros não utilizam o marketing pessoal, ocasionando ambiguidade e dilemas na profissão. ${ }^{17-18}$

\section{MATERIAL E MÉTODOS}

Por meio de revisão integrativa da literatura, buscou-se identificar o uso do marketing na enfermagem. Conforme preconizado, para o uso deste método, seguiu-se criteriosamente as seguintes etapas: identificação do problema e da questão de pesquisa, estabelecimento de critérios de inclusão e exclusão dos estudos, definição das informações a serem extraídas dos estudos selecionados, leitura e releitura dos estudos selecionados, identificação das informações relacionadas ao objetivo do estudo, e interpretação e síntese dos resultados encontrados. ${ }^{19}$ 
A questão norteadora elaborada para a seleção dos artigos desse estudo foi: como o marketing vem sendo utilizado pela enfermagem? Para a realização da busca foram definidos os seguintes descritores, de acordo com o catálogo da Bireme: enfermagem e marketing; nursing and marketing. As bases de dados consultadas foram a Medical Literature Analysis and Retrieval System on line (MEDLINE), Literatura Latino-Americana e do Caribe em Ciências da Saúde (LILACS), SciELO e Web of Science.

Utilizou-se como critérios de inclusão artigos disponíveis na íntegra, publicados no período de 2000 a 2010, nos idiomas português e inglês, nas bases de dados mencionadas, excluindo-se todos os que não atendessem a tais critérios.

Foram encontrados 91 artigos. Após leitura exaustiva dos títulos e resumos desses artigos, foram selecionadas as publicações disponíveis na íntegra, nos idiomas inglês e português, no período de 2000 a 2010, que estavam relacionadas ao tema pesquisado. Dessa forma, dentre os 91 artigos inicialmente encontrados, foram selecionados aqueles que abordavam diretamente o uso do marketing pela enfermagem. A análise dos artigos selecionados foi realizada após sua leitura na íntegra e com o auxílio de um instrumento de coleta de dados que contempla dados relacionados à identificação de autoria, ano e periódico de publicação, delineamento metodológico, assunto estudado, principais resultados e conclusões. ${ }^{20}$

Os estudos foram analisados e classificados em seis níveis de evidência, descritos na literatura por: nível I - estudos relacionados à metanálise de múltiplos estudos controlados; nível II - estudos experimentais individuais; nível III - estudos quase-experimentais, como ensaio clínico não randomizado, grupo único pré e pós-teste, além de séries temporais ou caso-controle; nível IV - estudos não experimentais, como pesquisa descritiva, correlacional e comparativa, com abordagem qualitativa e estudos de caso; nível V - dados de avaliação de programas e obtidos de forma sistemática; e nível VI - opiniões de especialistas, relatos de experiência, consensos, regulamentações e legislações. ${ }^{21}$

\section{RESULTADOS}

Foram selecionados oito artigos que abordavam diretamente o uso do marketing pela enfermagem, atendendo os critérios de inclusão. Destes, quatro relatam a opinião de especialistas, consensos ou relatos de experiência, caracterizando-se como de nível de evidência VI; três são estudos descritivos, caracterizados com nível de evidência IV; e um foi resultado dos dados de avaliação de um programa, classificado no nível de evidência $V$, o que demonstra que quando relacionamos marketing e enfermagem, encontramos um pequeno contingente de artigos, com baixo delineamento no nível de pesquisa.

O quadro 1 apresenta a sistematização dos artigos, segundo ano de publicação, autoria, periódico, país de publicação e estratégia de marketing utilizada na enfermagem.

Quadro 1 - Estudos que relacionam marketing e enfermagem no período de 2000-2010, de acordo com ano de publicação, autoria, título do periódico, país de publicação e estratégia de marketing utilizada para aumentar a visibilidade da enfermagem

\begin{tabular}{|c|c|c|c|c|}
\hline $\begin{array}{c}\text { Autor e ano de } \\
\text { publicação }\end{array}$ & Desenho do estudo & Periódico & $\begin{array}{c}\text { País de } \\
\text { publicação }\end{array}$ & Estratégia de marketing \\
\hline $\begin{array}{c}\text { Whitchead D } \\
2000\end{array}$ & Opinião de especialista & J Advanced Nursing & Inglaterra & $\begin{array}{c}\text { Uso dos meios de } \\
\text { comunicação em massa }\end{array}$ \\
\hline $\begin{array}{c}\text { Moura GMSS } \\
2003\end{array}$ & Opinião de especialista & $\begin{array}{l}\text { Rev Gaucha de } \\
\text { Enfermagem }\end{array}$ & Brasil & $\begin{array}{l}\text { Satisfação do cliente/ } \\
\text { paciente }\end{array}$ \\
\hline $\begin{array}{c}\text { Boyington AR, Wilson } \mathrm{DL}^{24} \\
2006\end{array}$ & Descritivo & Nursing Research & EUA & $\begin{array}{c}\text { Uso dos meios de } \\
\text { comunicação em massa }\end{array}$ \\
\hline $\begin{array}{c}\text { Boylston } \mathrm{J}^{25} \\
2008 \\
\end{array}$ & Descritivo & $\begin{array}{c}\text { Journal of } \\
\text { Professional Nursing }\end{array}$ & EUA & $\begin{array}{c}\text { Satisfação do cliente/ } \\
\text { estudante de enfermagem }\end{array}$ \\
\hline $\begin{array}{c}\text { Dion K, Smolenski M }{ }^{26} \\
2008 \\
\end{array}$ & Opinião de especialista & Imprint & EUA & Portfólio \\
\hline $\begin{array}{c}\text { Chang CS, Chang } \mathrm{HC}^{27} \\
2009 \\
\end{array}$ & Descritivo & $\begin{array}{c}\text { Journal of Advanced } \\
\text { Nursing }\end{array}$ & EUA & Endomarketing \\
\hline $\begin{array}{c}\text { Gentil RC }{ }^{18} \\
2009\end{array}$ & Opinião de especialista & Rev Bras Enferm & Brasil & Produção científica \\
\hline $\begin{array}{c}\text { Spear } \mathrm{HJ}^{28} \\
2010\end{array}$ & $\begin{array}{l}\text { Avaliação de } \\
\text { programa }\end{array}$ & J Christ Nurs & EUA & $\begin{array}{c}\text { Uso dos meios de } \\
\text { comunicação em massa }\end{array}$ \\
\hline
\end{tabular}


Dentre os oito estudos selecionados e analisados, observa-se que diferentes estratégias de marketing são utilizadas pela enfermagem para aumentar a visibilidade da profissão, visando à satisfação de seus clientes (internos e externos) e considerando os diferentes ambientes em que a enfermagem atua. Embora de maneira tímida e aprazada, foi possível identificar que a enfermagem tem recorrido ao recurso do marketing na profissão, utilizando os meios de comunicação em massa, a satisfação do cliente, o uso dos portfólios, o endomarketing e a produção científica. Ressalta-se, ainda, que na área da saúde é significativa a utilização do marketing social. O marketing social, que emergiu na década de 70, pode ser utilizado em campanhas, causas, ideias e comportamentos sociais, como projeto de implementação e controle de programas para aumentar a aceitação de uma ideia ou de uma prática social num determinado grupo-alvo.

Dentre os estudos analisados, um deles identificou, no site de 50 instituições hospitalares, que a maior parte deles não divulga a profissão, e que a única menção que fazem a ela é na divulgação de vagas de emprego. ${ }^{24}$

Por outro lado, foi demonstrado que, na enfermagem, conceitos de endomarketing são efetivos, uma vez que aliam a motivação ao marketing interno e educação, estando ainda diretamente ligados à diminuição da rotatividade dos funcionários dentro das organizações. ${ }^{27}$

Além do endomarketing, uma estratégia que vem sendo utilizada para a apresentação, o acompanhamento e a avaliação do profissional com julgamento positivo é o uso do portfólio eletrônico (registro de todas as atividades ocorridas ao longo da vida, com análise crítica e avaliação de maneira sistematizada).

Ainda, a divulgação de conhecimentos científicos, tecnológicos e humanos, apreendidos e desencadeados ao longo do desenvolvimento histórico da enfermagem, merece destaque também como estratégia de marketing. ${ }^{18}$

Dessa forma, cada um dos estudos, com as suas especificidades, contribui com informações sobre a possibilidade de incremento de estratégias de marketing pela enfermagem, inserindo conhecimentos de outras disciplinas e integrando-os à esta área.

\section{DISCUSSÃO}

Não se sabe ao certo quando surgiu a primeira ideia relacionada ao marketing; alguns atribuem o fato às primeiras relações de troca estabelecidas, outros à publicação da obra de Adam Smith "A riqueza das nações", e ainda, como uma corrente mais forte entre os autores, atribui-se o surgimento do marketing, às necessidades desencadeadas pela Revolução Industrial, geradora de uma grande quantidade de todos os tipos de produtos, que nem sempre conseguiam ser consumidos. No entanto, foi somente a partir do século XX que essa atividade passou a ganhar força e aceitação nas empresas, evoluindo seu foco de tarefas para o cliente. A era do marketing foi impulsionada a partir do período pós-guerra (II Guerra Mundial), marcada pelo nascimento de muitas crianças e pela explosão de adolescentes ávidos pelo consumo. ${ }^{15,29}$

O marketing é definido como uma função organizacional e um conjunto de processos que cria, comunica e agrega valor e gerencia relacionamentos com o cliente, beneficiando dessa forma a organização e os seus stakeholders. Sua aplicação nas empresas tem sido efetiva nos últimos anos, todavia; as mudanças oriundas da globalização, da preocupação social com questões ecológicas, da dificuldade de inserção dos profissionais no mercado de trabalho, levaram a sua utilização, aos mais diversos segmentos da sociedade. ${ }^{30-31}$

O uso dos meios de comunicação em massa constitui importante ferramenta na promoção do marketing profissional, podendo transferir ao público uma imagem positiva ou negativa do profissional, dependendo da maneira como é utilizado. Os estudos demonstraram que no cuidado direto ao paciente, nas funções educativas, gerenciais e organizacionais, a enfermagem contribui e auxilia na formação de uma grande rede de relacionamentos. Contudo, é ainda importante que se identifiquem, pela profissão, diferentes parceiros para a organização e a promoção de campanhas publicitárias, e no uso de meios legais para o confronto de tal situação. ${ }^{22,28}$

A comunicação em massa é uma importante estratégia para promover a imagem do enfermeiro. Considera-se que a imaginação dos profissionais da área de marketing no Brasil e o sucesso das campanhas publicitárias realizadas em nosso país estão entre as mais criativas do mundo. ${ }^{29}$ É indispensável agregar fatores positivos a todas as oportunidades de uso da imagem da enfermagem para a população, seja nas campanhas de promoção a saúde, no uso da imagem da profissão em programas e séries televisivas, e nas campanhas promocionais de prestadores de serviço. Busca-se, então, agregar valor ao serviço oferecido e às relações, segundo a ótica do consumidor. ${ }^{29,32-33}$ 
Objetiva-se, assim, a mudança de comportamento e de atitudes, numa determinada população, que nem sempre necessita ser a beneficiária das mudanças. No cotidiano do trabalho da enfermagem, constantemente, presenciamos a utilização do marketing social dos serviços de saúde, nas campanhas por melhores hábitos de vida, na promoção da saúde. O marketing social pode ainda ser melhor explorado em distintas mídias.

A velocidade de mudanças ocasionadas com o advento da internet, tanto na profusão das informações, como na divulgação de produtos, têm sido decisivas para intensificação do comércio eletrônico. Num mundo onde grande parte da população tem acesso à internet e busca informações sobre produtos, é necessário que também a enfermagem faça uso desse recurso como ferramenta de divulgação da profissão.

Nos dias atuais, muitos serviços de saúde e estudos na área de enfermagem focam seu objeto de trabalho na qualidade e satisfação do cliente. ${ }^{23}$ Ao definir o cliente no contexto da enfermagem são muitas as possibilidades do uso da palavra. $\mathrm{O}$ cliente, na enfermagem, pode ser entendido como o paciente, a família, a comunidade, os prestadores de serviços, assim como muitos outros clientes que, direta ou indiretamente, são beneficiados pelo resultado do seu trabalho. ${ }^{25}$

No âmbito acadêmico, a enfermagem também deve se esforçar para demonstrar o seu valor. Nesse sentido, é prioritário que as universidades identifiquem nos estudantes e futuros egressos dos cursos de enfermagem, clientes que necessitam identificar nos profissionais de enfermagem, características humanas e éticas de profissionais, pelos quais gostariam de ser cuidados.

$\mathrm{O}$ endomarketing caracteriza-se, assim, por estratégias institucionais internas de marketing, que garantam motivação para que sejam mantidos nas empresas os melhores profissionais e os melhores serviços. Acredita-se que a boa impressão ocasionada pelo atendimento prestado aos usuários dos serviços pode contribuir para a valorização e o reconhecimento do todos os profissionais que tenham optado pelo seguimento da mesma carreira. ${ }^{15}$

Na promoção do eu enfermeiro, a apresentação da trajetória acadêmica e profissional deve ser demonstrada de forma clara e objetiva. Cabe aos profissionais serem zelosos ao elaborarem e atualizarem seus currículos. Esta ferramenta possibilita o acompanhamento de toda a trajetória do profissional e sua educação ao longo da vida e vem sendo utilizada como estratégia de recru- tamento, seleção e avaliação da prática clínica do profissional, possibilitando sua inserção em sociedades especializadas, associações de classe, entre outros. ${ }^{26}$

Esta estratégia converge diretamente para nossa reflexão sobre o possível reconhecimento profissional, por meio da divulgação científica e também da demonstração de sua aplicação e transformação da prática.

\section{CONCLUSÃO}

$\mathrm{O}$ marketing na enfermagem é um tema pouco explorado em pesquisas. Todavia, há perspectivas de avanços pelo interesse que o tema desperta e pelo reconhecimento de sua importância. Os artigos analisados abordaram, em sua maioria, o uso dos meios de comunicação em massa como estratégia de marketing da profissão, para promoção de saúde e imagem profissional, veiculada em meios como a internet e a televisão.

Os resultados encontrados neste estudo nos levam a sugerir que esforços sejam envidados para o desenvolvimento de investigações acerca da temática, produzindo evidências que possam ser exploradas pela profissão.

Aponta-se, por fim, para a necessidade de desenvolvermos marketing da profissão: 1) para atrair alunos e formar mais profissionais qualificados; 2) para reter esses profissionais, através do endomarketing e de mais valorização; 3 ) para instituir, por meio de políticas públicas, mecanismos indutores da valorização do enfermeiro pelas instituições, pelos governos e pela sociedade; e 4) para que evidências sejam exploradas e empregadas para conquista de espaços e vitórias pelas instituições de ensino, de serviço, associações de classe, sociedades científicas e órgãos representativos da profissão.

Para o alcance desses propósitos, necessitamos de líderes que conduzam movimentos, que alcancem conquistas através de argumentos baseados em evidência e de várias estratégias, inclusive o marketing.

Em suma, o marketing na enfermagem deve compor um projeto de política pública que ultrapasse uma visão corporativa para buscar o fortalecimento da legitimidade social da profissão.

\section{REFERÊNCIAS}

1. Rigoli F, Dussalt G. The interface between health sector reform and human resources in health. 
Human Resour Health [Internet] 2003 [acesso 2010 Jul 13]; 1(9). Disponível em: http:/ / www.humanresources-health.com/content/1/1/9

2. Dussault G, Souza LE. Strategic management on human resources in health services. University of Montreal. School of Medicine, Department of Health Administration. Summer School on Public Health; 2000.

3. World Health Organization. World health statistics. Geneva (CH): World Health Organization; 2010.

4. World Health Organization. The world health report, 2008: primary health care-now more than ever. Geneva $(\mathrm{CH})$ : World Health Organization; 2008.

5. World Health Organization. Resolution WHA 54-12 Strenghtening nursing and midwifery. In: Fiftyfourth World Health Assembly, 2001 May 14-22. Geneva (CH): World Health Organization; 2001.

6. World Health Organization. Strategic directions for strengthening nursing and midwifery services, 20022008. Geneva $(\mathrm{CH})$ : World Health Organization; 2002.

7. World Health Organization. Resolution WHA 30.48 The role of nursing/midwifery personnel in primary health care teams. In: Thirtieth World Health Assembly, 1977 May 19. Geneva (CH): World Health Organization; 1977.

8. World Health Organization. Resolution WHA 36.11 The role of nursing/midwifery personnel in the Strategy for Health for All. In: Thirty-sixth World Health Assembly, Geneva (CH): World Health Organization; 1983. 13 May.

9. World Health Organization. Resolution WHA 42.27. Strengthening nursing/midwifery in support of the strategy for health for all. In: Forty-second World Health Assembly, Geneva (CH): World Health Organization; 1989. 19 May.

10. World Health Organization. Resolution WHA 45.5. Strengthening nursing/midwifery in support of strategies for health for all. In: Forty-fifth World Health Assembly, Geneva (CH): World Health Organization; 1992. 11 May.

11. World Health Organization. Resolution WHA 49.1. Strengthening nursing and midwifery. In: Fortyninth World Health Assembly, Geneva (CH): World Health Organization;1996. 23 May.

12. World Health Organization. Resolution WHA 54.12. Strengthening health services delivery. In: Fifty-fourth World Health Assembly, Geneva (CH): World Health Organization; 2001. 27 April.

13. World Health Organization. Resolution WHA 59.23. Rapid scaling up of health workforce production. In: Fifty-ninth World Health Assembly, Geneva $(\mathrm{CH})$ : World Health Organization; 2006. 22-27 May.

14. World Health Organization. Resolution WHA 59.27. Strengthening nursing and midwifery. In: Fifty- ninth World Health Assembly, Geneva (CH): World Health Organization; 2006. 22-27 May.

15. Fraga NEB, Mattos CE, Cassa GA. O marketing profissional e suas interfaces: a valorização do bibliotecário em questão. Perspect Ciênc Inf. 2008 Mai-Ago; 13(2):148-67.

16. Pereira ES, Filho GAL. A influência do marketing no perfil do profissional contábil. Contab Vista Rev. 2003 Abr; 14(1):75-89.

17. Luchesi LB, Santos CB. Enfermagem: o que esta profissão significa para adolescentes. Uma primeira abordagem. Rev Latino-am Enfermagem [online]. 2005 Mar-Abr [acesso 2010 Out 4]; 13(2). Disponível em: http://www.scielo.br/scielo.php?script=sci_ar ttest\&pid=S014-11692005000200005

18. Gentil RC. O enfermeiro não faz marketing pessoal: a história explica por quê? Rev Bras Enferm. 2009 Nov-Dez; 62(6):916-8.

19. Mendes KDSM, Silveira RCCP, Galvão CM. Revisão integrativa: método de pesquisa para a incorporação de evidências na saúde e na enfermagem. Texto Contexto Enferm. 2008 Out-Dez; 17(4):758-64.

20. Ursi ES, Galvão CM. Prevenção de lesões de pele no perioperatório: revisão integrativa da literatura. Rev Latino-am Enfermagem. 2006 Jan-Fev; 14(1):124-31.

21. Stetler CB, Morsi D, Rucki S, Broughton S, Corrigan $B$, Fitzgerald J, et al. Utilization-focused interative reviews in a nursing service. Appl Nurs Res. 1988 Nov; 11(4):195-206.

22. Whitehead D. Using mass media within healthpromotion practice: a nursing perspective. J Adv Nurs. 2000 Oct; 32(4):807-16.

23. Chicago. American Marketing Association. Definition of marketing [Internet]. Illinois; 2007. [acesso 2010 Ago 20]. Disponível em: http:// www.marketingpower.com/AboutAMA/Pages/ AMA\%20Publications/AMAPublications.aspx

24. Boyington AR, Jones CB, Wilson DL. Buried Alive: the presence of nursing on hospital web sites. Nurs Res. 2006 Mar-Apr; 55(2):103-9.

25. Boylston MT, Jackson C. Adult student satisfaction in an accelerated RN-to-BSN Program: a follow-up study. J Prof Nurs. 2008 Sep-Oct; 24(5):285-95.

26. Dion K, Smolenski MC. Reinventing your resume: electronically documenting your career. Imprint. 2008 Sep-Oct; 55(4):56-60.

27. Chang CS, Chang HC. Perceptions of internal marketing and organizational commitment by nurses. J Adv Nurs. 2009 Jan; 65(1):92-100.

28. Spear HJ. TV nurses: promoting a positive image of nursing? J Christ Nurs. 2010 Oct-Dec; 27(4):318-21.

29. Cobra M. Um resumo do percurso do marketing brasileiro. Revista FAE BUSINESS. 2002 Dez; 4:28-32.

30. Chicago. American Marketing Association. Definition of marketing [Internet]. Illinois; 2007. [acesso 2010 Ago 20]. Disponível em: http:// 
www.marketingpower.com/AboutAMA/Pages/ AMA\%20Publications/AMAPublications.aspx

31. Faria A. Marketing: a retórica do marketing. GVexecutivo. 2008 Mai-Jun; 7(3):44-7.

32. Kotler P, Hayes T, Bloom PN. Marketing de serviços profissionais: estratégias inovadoras para impulsionar a sua atividade, a sua imagem e os seus lucros. São Paulo (SP): Manole; 2002.

33. Silva EC, Mincioti SA. Marketing ortodoxo, societal e social: as diferentes relações de troca com a sociedade. RBGN. 2005 Abr; 7(17):15-22. 